> Les cellules souches embryonnaires pluripotentes (દS) ont des propriétés de prolifération et de différenciation qui font d'elles un outil prometteur pour la thérapie cellulaire. Malgré la légitime controverse sur les nombreuses questions éthiques que cette technologie suscite, et en dépit de ses potentiels dans des domaines aussi variables que la thérapie cardiaque, pulmonaire ou hépatique, ou la régénération osseuse et articulaire, c'est dans le traitement des maladies cérébrales que la technique ouvre les perspectives les plus intéressantes. De très nombreux travaux réalisés sur les cellules ES rapportent une régénération du cerveau et de la moelle épinière chez des rongeurs et la réparation des problèmes cognitifs causés lors de dommages apportés aux tissus lésés. Cet article offre une synthèse des avancées récentes dans le domaine de la manipulation des cellules souches (murines) en vue de sélectionner des populations de neurones, astrocytes et oligodendrocytes. $\varepsilon$ n parallèle, nous mettons l'accent sur les similitudes frappantes qui existent dans les programmes génétiques mis en œuvre au cours du développement embryonnaire in utero et au cours de la différenciation in vitro des cellules. <

\title{
Différenciation \\ neurale \\ des cellules \\ souches \\ embryonnaires
}

Michèle Cazillis, Vincent LeLièvre, Pierre Gressens

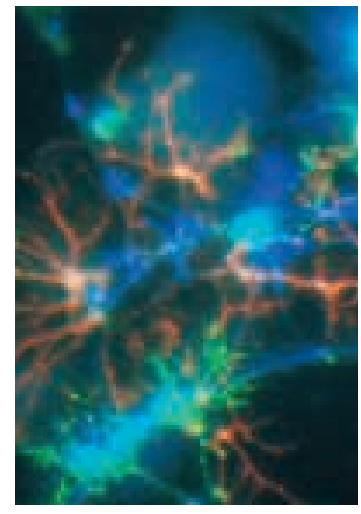

naire. Expérimentalement, les cellules ES possèdent un potentiel dans les domaines de la thérapie cardiaque, pulmonaire, hépatique ou encore de la régénération osseuse et articulaire [3], mais c'est dans le traitement des maladies cérébrales dégénératives que la technique ouvre ses perspectives les plus intéressantes $[4,5]$. Dans cet article, nous nous focaliserons sur la différenciation neurale des cellules souches embryonnaires murines ES, en suivant l'idée selon laquelle les cellules ES se différencient in vitro selon un programme génétique similaire à celui qui régit le développement embryonnaire in utero.

Les cellules souches embryonnaires murines $\varepsilon S$ sont isolées directement en culture à partir de la masse cellulaire interne (ICM) d'embryons âgés de 3,5 jours (blastocystes) [1, 2]. Elles sont pluripotentes et capables de se différencier in vivo dans tous les organes de l'embryon et, in vitro, dans la plupart des types cellulaires, à l'exception des tissus extra-embryonnaires. Les résultats actuellement disponibles indiquent que les cellules ¿S se différencient en suivant un programme génétique comparable (en termes de molécules, voies de signalisation, étapes et chronologie) à celui des cellules souches neurales au cours du développement embryon-

Article reçu le 19 juillet 2004 et accepté le 17 décembre 2004.

\section{Aspects techniques de la différenciation des cellules ES}

Deux méthodes principales sont utilisées pour différencier les cellules $\varepsilon S$ en cellules neurales. La première fait appel à la formation des corps embryoïdes, qui développent les trois feuillets germinatifs. Ils représentent un succédané, in vitro, des embryons au stade périimplantatoire: les cellules produisent une population mixte contenant en proportion variable des neurones et des cellules gliales (astrocytes et oligodendrocytes). La seconde méthode consiste à cultiver les cellules ES en milieu sans sérum, conditionné ou non par différents morphogènes: les cellules ES produisent alors des 
populations quasi homogènes de neurones ou de cellules gliales. L'ensemble des résultats montrent que les cellules $E S$, in vitro, produisent et répondent aux différents signaux responsables de la différenciation neurale de l'embryon (Figure 1).

\section{Pluripotentialité des cellules ES}

Les cellules $\varepsilon S$, toutes premières cellules souches de l'embryon, sont capables de s'autorenouveler, c'est-à-dire de se reproduire à l'infini sans perdre leur potentialité. Ce phénomène nécessite l'activation de la voie STAT et des facteurs de transcription NANOG et OCT (Figure 2).

La voie de transduction STAT, activée par l'interaction de différents signaux extracellulaires (IL-6 et LIF, par exemple) avec un récepteur commun (Gp130), conduit à l'activation du cycle cellulaire et au maintien de l'état indifférencié. Chez l'embryon, l'inactivation génique de stat3 ou de gp130 est létale au stade $\varepsilon 6$ (précoce) de développement $[6,7]$, confirmant le rôle prépondérant de la voie STAT3 dans le développement embryonnaire. En revanche, les souris invalidées pour le gène codant pour le facteur LIF sont viables [8], ce qui suggère que d'autres facteurs endogènes régulent la voie STAT3 in vivo. In vitro, l'activation de STAT3 par LIF est suffisante pour assurer l'autorenouvellement des cellules ES; à l'inverse, en l'absence de LIF pour activer la voie STAT3, les cellules $E S$ se différencient spontanément $[6,9,10]$. De même, l'expres-

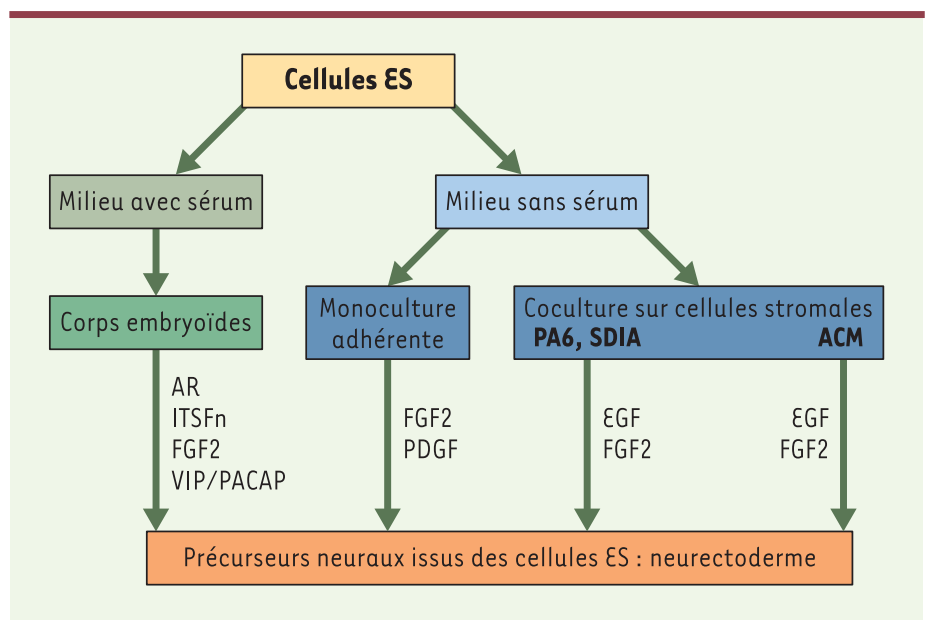

Figure 1. Différenciation des cellules $\varepsilon S$ en cellules neurales. Deux types de techniques de culture avec ou sans sérum permettent d'obtenir des cellules neurales à partir de cellules ES. En milieu avec sérum, les cellules se différencient en corps embryoïdes. Le milieu est conditionné soit par l'acide rétinoïque (AR) $[26,50]$, soit par le milieu ITSFn (contenant insuline, transferrine, sélénium et fibronectine) [4]. Deux neuropeptides, le VIP et le PACAP, induisent la différenciation des $\varepsilon S$ dans les corps embryoïdes [51]. En milieu sans sérum, les cellules \&S sont cultivées en monocouche [25] ou en coculture sur des cellules stromales. Les cellules PA6 produisent à leur surface cellulaire le SDIA, un principe actif qui induit la différenciation neurale des cellules [52]. La coculture en présence d'astrocytes (ACM) induit également la neuralisation [11]. Les facteurs EGF et FGF2 favorisent la prolifération des précurseurs neuraux issus des cellules ES. Le PDGF permet, quant à lui, d'achever la différenciation gliale. sion ectopique d'un mutant dominant négatif de STAT3 déclenche la différenciation cellulaire. Cependant, lorsque les cellules ES sont établies en milieu sans sérum (avec les suppléments N2 et B27 qui conditionnent le milieu de culture des cellules neuronales), des voies alternatives à LIF/STAT3 sont requises pour induire l'autorenouvellement des cellules ES, et plus particulièrement pour inhiber la différenciation neurale [11] (Figure 2). LIF seul augmente le nombre de cellules \&S non différenciées. La combinaison de LIF avec BMP4 ou BMP2 (bone morphogenic proteins) augmente le taux d'autorenouvellement des cellules ES non différenciées [11]. La combinaison de LIF et BMP permet de maintenir le potentiel de différenciation: leur carence entraîne la différenciation neurale. Cependant, l'effet de la voie BMP/SMAD sur l'autorenouvellement reste assujetti à l'état d'activation de la voie de signalisation de LIF.

Par ailleurs, deux facteurs de transcription à homéodomaine, NANOG et OCT4, semblent étroitement contrôler l'autorenouvellement et/ou la différenciation des cellules souches [11-13]. NANOG et OCT4 sont exprimés dans la masse cellulaire interne (ICM) et les cellules épiblastiques des embryons préimplantés, et disparaissent au cours du développement précoce (gastrulation).

La délétion partielle de nanog, qui provoque une réduction d'environ $50 \%$ du taux de NANOG produit dans les cellules $E S$ invalidées, se traduit par une différenciation multidirectionnelle soutenue [14]. À l'inverse, la surexpression de NANOG permet aux cellules \&S de s'autorenouveler en l'absence de LIF et de BMP [11]. NANOG est exprimé dans les embryons oct4 $4^{-/}$, suggérant qu'OCT4 et lui-même suivent des voies différentes [15].

OCT4 est exprimé tout d'abord dans l'ovocyte; son expression décline ensuite pendant les premiers clivages, puis réapparaît au stade $4-8$ cellules. II est confiné à I'ICM et à l'épiblaste, pour disparaître quand la différenciation apparaît, mais son expression persiste dans les cellules germinales (pluripotentes et/ou totipotentes) [11-13, 15]. Chez l'embryon de souris, l'invalidation permanente du gène oct4 empêche l'établissement de populations cellulaires pluripotentes et provoque l'arrêt du développement [13]. In vitro, selon son degré d'expression, 0ct4 gouverne trois destins différents des cellules ES: le taux normal d'expression est requis pour maintenir l'autorenouvellement des cellules દS; son augmentation favorise la formation de l'ectoderme primitif [15], puis de l'ectoderme en endoderme définitif et mésoderme; au contraire, la répression de OCT 4 induit la perte de la pluripotentialité et la dédifférenciation en trophectoderme. 


\section{Différenciation/spécification des cellules $\varepsilon S$ en précurseurs du neurectoderme}

La différenciation de l'ectoderme primitif en neurectoderme est à l'origine de la différenciation neurale. Les cellules sont alors orientées vers un destin neural par l'action centrale des BMP. Cette étape est un préalable à l'acquisition par les précurseurs neuraux de l'identité rostrocaudale, puis dorsoventrale. Malgré l'absence de structure tridimensionnelle, les résultats expérimentaux démontrent que la différenciation des cellules $\varepsilon S$ en culture dépend des mêmes stimulus morphogènes que ceux identifiés dans la mise en place des territoires nerveux embryonnaires in vivo.

\section{BMP4 et différenciation proneurale des cellules ES} In vivo, BMP4 participe à la différenciation neurale selon un modèle par défaut $[16,17]$ : crucial pour le choix entre un destin épidermique ou neural, BMP4 induit par son action directe la différenciation épidermique, tandis que tout événement l'inhibant conduit à la détermination du neurectoderme. Ce modèle par défaut, bien validé chez l'embryon de batracien et de poisson-zèbre, reste cependant controversé chez le poussin et la souris. L'inhibition de BMP4 a lieu soit au niveau de sa voie de signalisation, soit au niveau de la transcription où elle est régulée par les effets croisés de la signalisation de Wnt et des FGF [18]. En effet, les facteurs sécrétés Noggin, Chordin et Follistatin, inhibiteurs naturels de BMP4, voient leur expression régulée par Wnt et FGF; ainsi, le blocage de la voie de signalisation des FGF réduit la synthèse de Chordin et réprime la neuralisation de l'embryon de xénope. Enfin, l'acide rétinoïque $(A R)$, produit localement dans le cerveau embryonnaire et dont l'action est partiellement dépendante de la voie de signalisation de Wnt et des FGF, est requis pour établir la spécification neurale. En résumé, BMP4 induit par défaut la neuralisation et ses effets sont modulés par une boucle de régulation Wnt/FGF/AR; responsable de la spécification neurale (Figure 2), cette boucle interviendra également au moment de l'acquisition de l'identité dorsoventrale (voir plus loin). In vitro, les cellules $E S$, qui produisent BMP2 et BMP4, en concentrations très faibles, et les antagonistes Noggin et Chordin, acquièrent un phénotype neural en l'absence de BMP4 exogène [19]. L'ajout de Noggin ou Chordin au milieu de culture, ou leur surexpression constitutive dans les cellules ES, entraîne l'augmentation du nombre de colonies différenciées. L'effet inhibiteur de BMP4 sur la différenciation neurale a été plus directement démontré dans des lignées de cellules ES invalidées pour le gène Smad4, qui code pour un effecteur central de sa voie de signalisation. Dans des conditions de culture induisant la différenciation (SDIA), la présence d'une mutation invalidante du gène Smad4 induit la transition neurale des cellules ES sans influencer la multiplication cellulaire, puisque le nombre de colonies formées n'est pas affecté par les doses de LIF ou de sérum [19]. Ces observations témoignent que c'est aussi l'inhibition de BMP4 qui induit la différenciation neurale dans les cellules ES. Cette action de BMP4 sur la différenciation des $E S$ semble limitée dans le temps, de façon à coïncider avec son effet sur la gastrulation précoce de l'embryon [9, 20, 21].

La signalisation de Wnt intervient également dans la différenciation in vitro. Cette voie de signalisation est complexe et aboutit classiquement à l'accumulation nucléaire de $\beta$-caténine et la transactivation de gènes cibles. L'action de cette voie Wnt/ $\beta$-caténine sur l'induction neurale reste controversée et semble dépendre du modèle cellulaire et des facteurs présents localement. Certaines études montrent que l'inhibition de

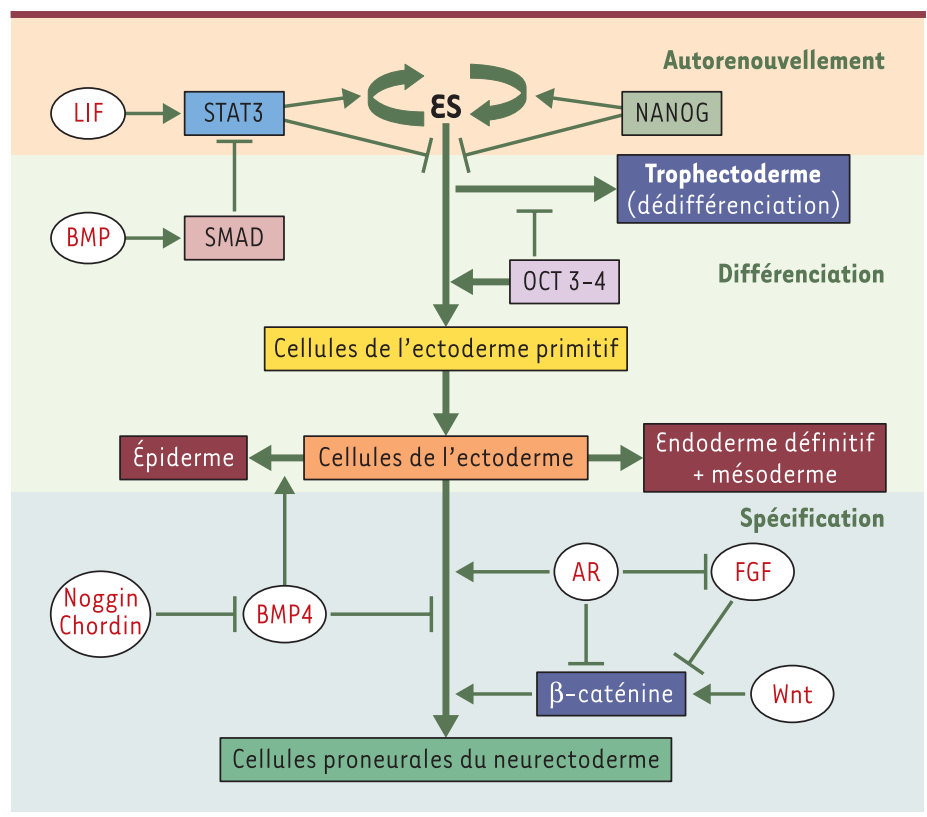

Figure 2. Pluripotentialité des cellules $\varepsilon S$ et induction de leur différenciation en cellules du neurectoderme. La prolifération des cellules ES est assurée par le facteur soluble LIF, au travers de l'activation de STAT3. De son côté, BMP active SMAD, qui inactive STAT3. NANOG intervient quant à lui en activant la prolifération des cellules \&S et en inhibant leur différenciation. L'inactivation de STAT3, en l'absence de LIF, induit la différenciation des cellules $\varepsilon S$ en cellules de l'ectoderme primitif. En parallèle, OCT4, à une concentration basale, stimule l'autorenouvellement. Son absence entraîne en revanche la différenciation des cellules $E S$ en trophectoderme; à l'inverse, des doses élevées d'OCT4 induisent la différenciation des cellules ES en cellules de l'ectoderme primitif. L'inhibition de BMP4, grâce aux inhibiteurs naturels tels que Noggin et Chordin, induit la spécification neurale par défaut, au détriment de la différenciation épidermique. L'acide rétinoïque (AR) active la différenciation des cellules de l'ectoderme en neurectoderme au travers de deux mécanismes, l'un direct, l'autre via l'inhibition de la voie de signalisation Wnt/ $\beta$-caténine. Par ailleurs, l'acide rétinoïque inhibe aussi la voie de signalisation des FGF, connue pour sa régulation négative de la voie Wnt. 
la voie de Wnt à différents niveaux [22, 23] dans les cellules ES stimule la différenciation neurale et que l'activation de la voie WNT augmente l'expression régulée de BMP4 et BMP7, inhibiteurs spécifiques de la différenciation neurale [24]. Des études plus récentes démontrent, au contraire, l'effet neuralisant de l'activation de la $\beta$-caténine dans les cellules $\varepsilon S$, suggérant que l'action de Wnt implique l'activation de voies de signalisation multiples et antagonistes. L'AR, en réprimant la voie de signalisation par FGF, lève l'inhibition de la voie Wnt/ $\beta$-caténine et active en cascade l'expression de gènes neuralisants et la différenciation neuronale. Le traitement des cellules ES par le FGF peut cependant induire l'expression de marqueurs généraux de la différenciation neuronale comme Soxl [25]. Les FGF suffisent mais ne sont pas absolument nécessaires à la différenciation des cellules ES en culture; si leur présence assure la prolifération des cellules en différenciation, leur défaut induit la différenciation neurale [25]. Enfin, l'AR joue un rôle très important dans l'induction de la différenciation neurale des cellules ES (il a ainsi été beaucoup utilisé pour induire la différenciation neurale dans les corps embryoïdes et les cellules ES en culture [26]), même si ses mécanismes d'action ne sont pas exactement élucidés [27, 28]. Tous ces résultats indiquent que les cellules ES sont spécifiées en cellules proneurales selon des mécanismes comparables au développement embryonnaire in vivo. Ces précurseurs issus des cellules $\varepsilon S$ vont poursuivre leur différenciation et acquérir les caractéristiques de l'identité de position dans le tube neural.

\section{Acide rétinoïque et identité antéropostérieure des cellules proneurales}

L'acide rétinoïque est produit le long de l'axe rostrocaudal du cerveau embryonnaire où il intervient dans le patron des territoires des cellules précurseurs. In vitro, il est associé à la différenciation précoce des cellules \&S dans les corps embryoïdes, tout comme il intervient dans la spécification des cellules souches neurales dans l'embryon [20, 29]. Selon la dose d'AR utilisée, la différenciation terminale des $\varepsilon S$ en cellules neurales est facilitée. L'AR semble jouer un rôle complémentaire dans la spécification de l'identité de position rostrocaudale. In vitro, cet effet a été étudié en détail sur des cellules ES différenciées en corps embryoïdes [30]. L'action caudalisante de l'AR (apparition des marqueurs Hoxc5/6 au détriment de marqueurs plus antérieurs comme 0 tx2 et $\varepsilon n 1$ ) se combine aux effets de SHH pour obtenir des motoneurones [31]. À l'inverse, on obtient des cellules exprimant des marqueurs de cerveau antérieur (emxl, emx2, nkx2.1) en l'absence d'AR, et des cellules exprimant des marqueurs des neurones du cerveau moyen pour des doses faibles [27].

\section{Acquisition de l’identité dorsoventrale par les cellules proneurales}

BMP et SHH s'opposent pour produire, à partir des cellules souches, les populations neuronales qui déterminent l'axe dor- soventral de l'embryon. L'axe dorsoventral est spécifié essentiellement par les actions antagonistes de BMP4 et SHH. Les facteurs EGF, FGF8 et FGF2, ainsi que I'AR sont également requis. Les voies de signalisation de l'AR et du FGF interagissent dans la régulation de la spécification de l'axe rostrocaudal: l'inhibition de la voie de signalisation du FGF supprime ainsi l'expression du récepteur de I'AR (RAR). À l'inverse, la surexpression du récepteur RAR $\alpha$ restaure les effets du FGF, ce qui suggère que le récepteur de l'AR, RAR $\alpha 2$, est bien une cible directe de la voie de signalisation du FGF [32].

\section{BMP4 et dorsalisation des cellules proneurales}

Les cellules proneurales issues des cellules $E S$ se différencient en précurseurs des motoneurones et cellules de la crête neurale par l'action de BMP4. Dans l'embryon, le facteur BMP4 est sécrété par le plafond du tube neural. II diffuse selon un gradient le long du tube neural pour agir différentiellement, selon la dose et la position, sur l'identité dorsale des précurseurs neuronaux. Ces précurseurs se différencient séquentiellement en neurones, puis en précurseurs des cellules de la crête neurale [33]. L'action de BMP4, en fin de gastrulation, sur les cellules proneurales issues des cellules ES cultivées en milieu défini conduit à l'acquisition progressive des caractères propres aux neurones dorsaux et aux cellules de la crête neurale [30]. Ainsi, la présence de BMP4 augmente le nombre de cellules exprimant les marqueurs de la crête neurale (snail, slug, Msxl) au détriment des marqueurs ventraux (Nkx2.2 et HNF3 $\beta$ ). De plus, BMP4 induit, à partir des précurseurs de la crête neurale, la différenciation de neurones sensoriels (Brn3a/Peri) ou, pour une dose plus élevée, de neurones autonomes (TH/Peri). Ces résultats montrent, de nouveau, que BMP4 induit la différenciation des cellules \&S suivant un processus similaire à celui observé chez l'embryon (Figure 3).

\section{SHH et différenciation ventrale des cellules proneurales}

Sonic hedgehog induit la différenciation ventrale des cellules proneurales issues des cellules ES pour produire des motoneurones. Dans l'embryon de souris, SHH est produit par la notocorde, puis par le plancher du tube neural. Il diffuse selon un gradient de concentration dans la partie ventrale du tube neural pour permettre localement la différenciation de précurseurs ventraux $[16,17]$. La perte de SHH ou l'interruption de sa voie de signalisation produit la dorsalisation de l'embryon par expansion des territoires sous contrôle de BMP4. À l'inverse, l'expression constitutive de SHH réprime l'expression des territoires dorsaux [28].

Plusieurs études ont démontré que les corps embryoïdes dérivés des cellules \&S murines expriment Indian hedgehog $(\mathrm{IHH})$, I'un des trois membres de la famille Hedgehog, au niveau de l'endoderme viscéral externe, et les molécules en aval dans la cascade de signalisation (Patched et Glil) au niveau de l'endoderme interne [34]. L'extinction de l'expression d'IHH et de son coré- 
cepteur SMO dans les cellules \&S a démontré que l'activation de la voie de signalisation Hedgehog est requise pour la différenciation des cellules $\varepsilon S$ de souris par l'acide rétinoïque [35]. Le traitement séquentiel de cellules ES dont la neuralisation a été induite par culture sur les cellules stromales a permis de préciser la chronologie de l'intervention des facteurs dans l'obtention des différents neurones et cellules gliales. Ainsi, en présence de concentrations croissantes de $\mathrm{SHH}$, les cellules exprimant les marqueurs de la crête neurale et des neurones dorsaux (Pax7 et Mathl) diminuent et disparaissent au profit de l'apparition progressive de cellules exprimant les marqueurs ventraux (Nkx2.2 et HNF3 $\beta$ ). Plus tard, les marqueurs secondaires apparaissent, identifiant des populations plus spécialisées comme les motoneurones télencéphaliques ou des neurones brachiomoteurs et viscéraux [30] (Figure 3). Des techniques alternatives proposent l'ajout séquentiel des facteurs responsables de la différenciation sans apport exogène de BMP4 et $\mathrm{SHH}$. Ces techniques permettent d'orienter le processus selon des voies de neuralisation parallèles qui aboutissent à l'obtention des neurones dopaminergiques du cerveau moyen, des neurones sérotoninergiques du cerveau postérieur, des motoneurones de la moelle épinière et des neurones GABAergiques, ainsi que des astrocytes et des oligodendrocytes [36].

Enfin, l'expression du gène nurrl est requise pour la spécification et la différenciation des neurones dopaminergiques. Les progéniteurs neuraux obtenus à partir de cellules ES surexprimant le récepteur nucléaire Nurrl se différencient sélectivement en neurones dopaminergiques [37]. La présence de Nurrl potentialise également les effets de SHH et du FGF8 sur la différenciation neuronale des cellules ES [38]. À l'inverse, les souris partiellement invalidées pour Nurrl développent des troubles, en fonction de l'âge, du système dopaminergique [39]. Enfin, ces mêmes lignées ES développent des caractères fonctionnels de neurones dopaminergiques lorsqu'ils sont transplantés dans des cerveaux de rats présentant des troubles similaires à ceux observés chez les patients atteints de la maladie de Parkinson [38].

\section{Différenciation gliale (astrocytes et oligodendrocytes)}

Dans l'embryon, la différenciation gliale apparaît après la différenciation neuronale dans les zones ventrales et dorsales du tube neural. Elle est directement contrôlée par SHH et implique l'expression des facteurs de transcription Olig2 et $\mathrm{Nk} \times 2.2$; cependant, une voie alterne indépendante de $\mathrm{SHH}$ existe vraisemblablement [40]. Olig2 est initialement exprimé dans les progéniteurs oligodendrocytaires ventraux et, inversement, réprimé dans les neurones et les astrocytes. La différenciation oligodendrocytaire est en effet réduite et retardée chez les souris n'exprimant plus ce facteur de transcription. Par ailleurs, les progéniteurs exprimant Olig2 sont capables de myéliniser des

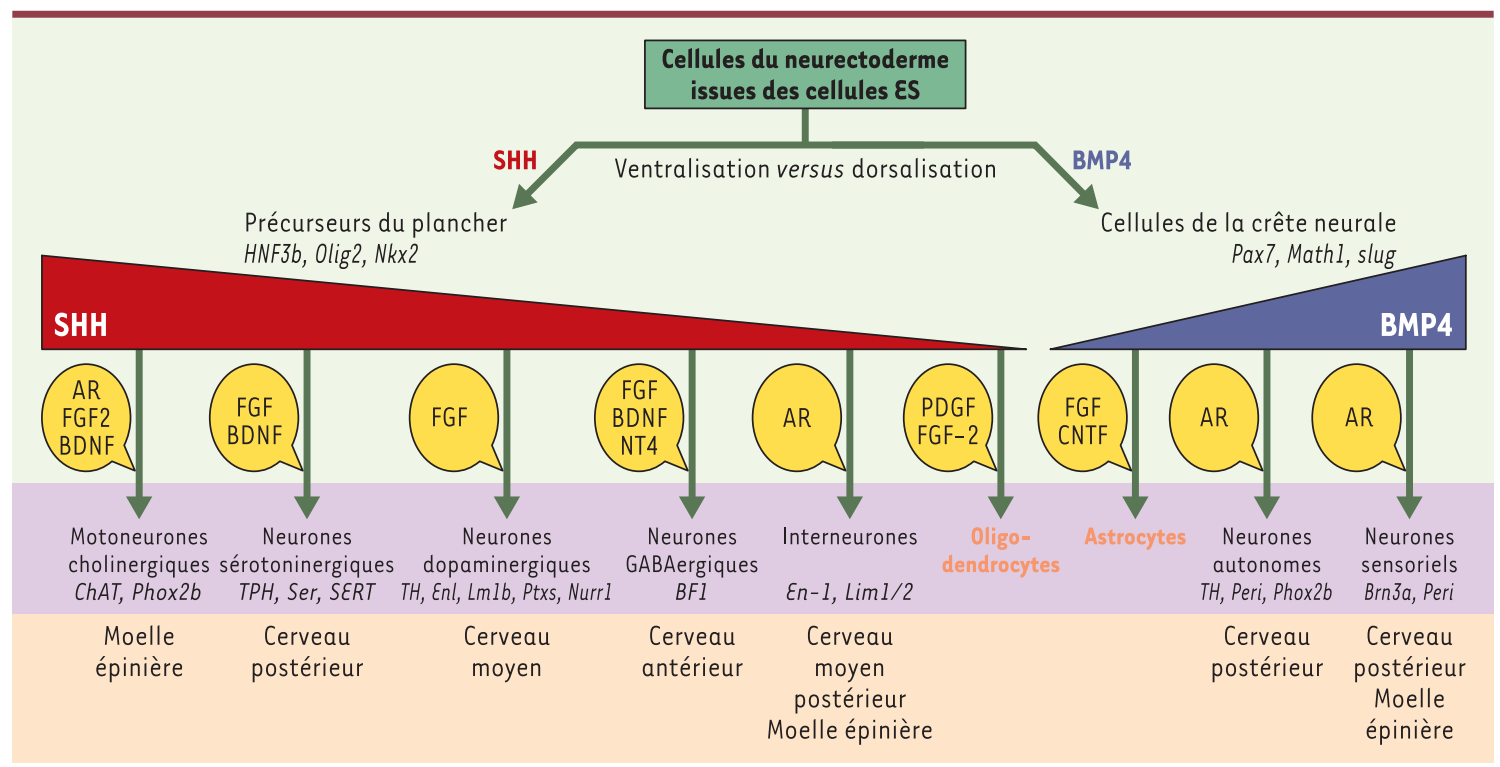

Figure 3. Différenciation des cellules proneurales issues des cellules ES dans les différents types de cellules neurales. L'action opposée de SHH et BMP4 sur les cellules proneurales conduit à la production de cellules précurseurs du plancher et de cellules de la crête neurale. Les marqueurs spécifiques des différents types cellulaires sont en italiques. Les types de neurones sont placés selon le gradient de concentration qui permet l'acquisition de leur identité. La localisation embryonnaire correspondant à l'identité acquise par les cellules issues des cellules ES est indiquée sous les différents neurones. Le schéma montre que la différenciation des cellules $\varepsilon S$ en neurones reproduit la différenciation spatiotemporelle obtenue au cours du développement embryonnaire in vivo. Les facteurs de différenciation sélective (FGF, BDNF, NT4, PDGF, CNTF et AR) sont indiqués dans les bulles jaunes. 
axones en culture et, in vivo, dans la moelle épinière endommagée $[41,42]$. L'expression du marqueur Nkx2.2 participe à la spécification oligodendrocytaire, puisque l'apparition d'oligodendrocytes matures est fortement réduite sur l'ensemble de l'axe rostrocaudal chez les animaux mutants dénués de Nkx2.2, tandis que la population astrocytaire reste inchangée. La différenciation astrocytaire apparaît également au niveau dorsal du tube neural sous l'influence de BMP4. À ce niveau, les progéniteurs semblent incapables de donner naissance à des populations oligodendrocytaires, suggérant une origine distincte de celle des progéniteurs oligo-astrocytaires ventraux.

Dans certaines conditions de culture, les cellules $E S$ se différencient en cellules gliales [41, 43]. Les corps embryoïdes peuvent produire des cellules astrogliales, oligodendrogliales et microgliales (visualisées respectivement par les marqueurs GFAP, 04 et 5 C6 ou galectin-3) selon un processus séquentiel qui rappelle la dynamique des mécanismes mis en jeu in utero [44]. Ainsi, les cellules ES surexprimant de façon constitutive Olig2 produisent des oligodendrocytes et des motoneurones [45]. Enfin, l'ajout de FGF2 sur les corps embryoïdes induit la co-expression d'0lig2 et du récepteur du PDGF dans $12 \%$ des cellules; cette proportion de cellules doublement marquées passe à $85 \%$ en 48 heures lorsque SHH et FGF sont tous deux ajoutés, confirmant l'action conjointe de SHH et FGF dans la genèse oligodendrocytaire. Enfin, l'action séquentielle et temporelle de ces facteurs en culture a été démontrée pour l'obtention en nombre d'astrocytes ou d'oligodendrocytes [36] (Figure 3).

\section{Conclusions}

L'ensemble des travaux présentés dans cet article démontrent que la différenciation neurale des cellules ES en culture se déroule selon un programme génétique similaire à celui observé chez l'embryon. En l'absence d'une structure tridimensionnelle, les cellules ES sont a priori capables de donner naissance non seulement à toutes les populations neuronales (motrices, sensorielles et associatives) produites in vivo chez la souris, mais aussi aux lignées oligo-astrogliales. La culture des cellules ES en présence de signaux extracellulaires, identifiés comme morphogéniques chez l'embryon, activent des programmes de spécification et d'identité de position selon un programme spatiotemporel similaire à celui mis en place dans l'embryon. Dans ce contexte, l'utilisation des cellules souches ES natives ou prédifférenciées dans le traitement de modèles animaux des maladies neurodégénératives humaines apparaît comme une avancée décisive. Ainsi, de nombreux travaux récents rapportent l'implantation réussie de cellules souches dans le cerveau de souris présentant des conditions pathologiques reproduisant certains troubles neurologiques observés chez l'homme [29]. $\diamond$

\section{SUMMARY}

Neural differentiation of murine embryonic stem cells $\varepsilon S$

Pluripotent murine embryonic stem (દS) cells can differentiate into all cell types both in vivo and in vitro. Based on their capability to proliferate and differentiate, these ES cells appear as a very promising tool for cell therapy. The understanding of the molecular mechanisms underlying the neural differentiation of the ES cells is a pre-requisite for selecting adequately the cells and conditions which will be able to correctly repair damaged brain and restore altered cognitive functions. Different methods allow obtaining neural cells from ES cells. Most of the techniques differentiate ES cells by treating embryoid bodies in order to keep an embryonic organization. More recent techniques, based on conditioned media, induce a direct differentiation of $\varepsilon S$ cells into neural cells, without going through the step of embryonic bodies. Beyond the fact that these techniques allow obtaining large numbers of neural precursors and more differentiated neural cells, these approaches also provide valuable information on the process of differentiation of $E S$ cells into neural cells. Indeed, sequential studies of this process of differentiation have revealed that globally $\varepsilon S$ cells differentiating into neural cells in vitro recapitulate the molecular events governing the in vivo differentiation of neural cells. Altogether these data suggest that murine ES cells remain a highly valuable tool to obtain large amounts of precursor and differentiated neural cells as well as to get a better understanding of the mechanisms of neural differentiation, prior to a potential move towards the use of human $\varepsilon S$ cells in therapy. $\diamond$

\section{RÉFÉRENCES}

1. Evans MJ, Kaufman MH. Establishment in culture of pluripotential cells from mouse embryos. Nature 1981; 292: 154-6.

2. Martin GR. Isolation of a pluripotent cell line from early mouse embryos cultured in medium conditioned by teratocarcinoma stem cells. Proc Natl Acad Sci USA 1981; 78: 7634-8.

3. Gerecht-Nir S, Itskovitz-Eldor J. Cell therapy using human embryonic stem cells. Transpl Immunol 2004; 12 : 203-9.

4. Fluckiger AC, Dehay C, Savatier P. Embryonic stem cells and cell replacement therapies in the nervous system. Med Sci (Paris) 2003; 19:699-708.

5. McKay RD. Stem cell biology and neurodegenerative disease. Philos Trans R Soc Lond B Biol Sci 2004 ; 359: 851-6.

6. Takeda K, Noguchi K, Shi W, et al. Targeted disruption of the mouse Stat3 gene leads to early embryonic lethality. Proc Natl Acad Sci USA 1997; 94: 3801-4.

7. Li M, Sendtner M, Smith A. Essential function of LIF receptor in motor neurons. Nature $1995 ; 378: 724-7$.

8. Stewart CL, Kaspar P, Brunet LJ, et al. Blastocyst implantation depends on maternal expression of leukaemia inhibitory factor. Nature 1992; 359: 76-9.

9. O'Shea KS. Self-renewal versus differentiation of mouse embryonic stem cells. Biol Reprod 2004; 71 : 1755-65.

10. Nichols J. Introducing embryonic stem cells. Curr Biol 2001; 11: R503-5.

11. Mitsui K, Tokuzawa $Y$, Itoh $\mathrm{H}$, et al. The homeoprotein Nanog is required for maintenance of pluripotency in mouse epiblast and ES cells. Cell 2003; 113: 631-42.

12. Niwa H. Molecular mechanism to maintain stem cell renewal of $\varepsilon S$ cells. Cell Struct Funct $2001 ; 26: 137-48$.

13. Nichols J, Zevnik B, Anastassiadis K, et al. Formation of pluripotent stem cells in the mammalian embryo depends on the POU transcription factor Oct4. Cell 1998; 95: 379-91.

14. Hatano SY, Tada M, Kimura H, et al. Pluripotential competence of cells associated with Nanog activity. Mech Dev 2005; $122: 67-79$.

15. Shimozaki K, Nakashima K, Niwa H, et al. Involvement of $0 \mathrm{ct} 3 / 4$ in the enhancement of neuronal differentiation of $\varepsilon S$ cells in neurogenesis-inducing cultures. Development 2003; 130: 2505-12. 


\section{GLOSSAIRE}

5 C6 : anticorps 5 C6 spécifique du collagène de type IV

ACM: astrocyte-conditioned medium

BDNF : brain-derived neurotrophic factor

BF1: brain factor-1

BMP: bone morphogenic protein

Brn3a: brain-specific homeobox/POU domain protein 3

ChAT: choline acétyl transférase

CNTF : ciliary neurotrophic factor

EGF : epidermal growth factor

emxl et-2: empty spiracles homolog $1 / 2$

En 1 : engrailed gene 1

FGF2, -8: fibroblast growth factor 2, -8

GFAP: glial fibrillary acidic protein

Gli1: GLI-Kruppel family member 1

HNF3 $\beta$ : hepatocyte nuclear factor $3 \beta$

Hox: famille de gènes homeobox

IHH: Indian hedgehog

IL-6: interleukine-6

LIF : leukemia-inhibiting factor

Lim1/2: Lim domain gene 1/2

Math1: mouse basic helix-loop-helix transcription homolog of the drosophila astral gene

Msxl: msh-like 1

NCAM: neural cell adhesion molecule

nkx2.1 et -2.2: TTF-1: hybrid transcription factor 1 and 2

NT4 : neurotrophin 4

Nurrl : nuclear transcription factor receptor 1

04 : marqueur oligodendrocytaire

0ct4 (3/4): POU-domain transcription factor

Olig2: oligodendrocyte lineage transcription factor 2

0tx2: orthodenticle homolog 2

PA6: stromal cells derived from skull bone marrow

PACAP: pituitary adenylate cyclase-activating peptide

Pax7 : paired box gene 7

PDGF : platelet-derived growth factor

Peri : peripherin peripheral autonomic cell

Phox $2 \mathrm{~b}$ : paired mesoderm homeobox protein $2 b$

Ptx: pentraxin

SDIA: stromal cell-derived inducing activity

Ser: sérotonine

SERT: transporteur de la sérotonine

SHH: Sonic hedgehog

SMAD: homologue de MAD: mothers against DPP (decapentaplegic)

Soxl: SRy-box containing gene 1

STAT3 : signal transducer and activator of transcription 3

TH: tyrosine hydroxylase

TPH: tryptophane hydroxylase

VIP: vasoactive intestinal peptide

Wnt: wingless-Int
16. Panchision DM, McKay RD. The control of neural stem cells by morphogenic signals. Curr Opin Genet Dev 2002; 12: 478-87.

17. Jessell TM. Neuronal specification in the spinal cord: inductive signals and transcriptional codes. Nat Rev Genet 2000; 1: 20-9.

18. Wilson SI, Edlund T. Neural induction: toward a unifying mechanism. Nat Neurosci 2001; 4 (suppl): 1161-8.

19. Tropepe V, Hitoshi S, Sirard C, et al. Direct neural fate specification from embryonic stem cells : a primitive mammalian neural stem cell stage acquired through a default mechanism. Neuron $2001 ; 30: 65-78$

20. Czyz J, Wobus A. Embryonic stem cell differentiation: the role of extracellular factors. Differentiation 2001; 68: 167-74.

21. Coucouvanis $\varepsilon$, Martin GR. BMP signaling plays a role in visceral endoderm differentiation and cavitation in the early mouse embryo. Development $1999 ; 126$ : 535-46.

22. Aubert J, Dunstan $\mathrm{H}$, Chambers I, et al. Functional gene screening in embryonic stem cells implicates Wnt antagonism in neural differentiation. Nat Biotechnol 2002; $20: 1240-5$.

23. Kielman MF, Rindapaa M, Gaspar C, et al. Apc modulates embryonic stem-cell differentiation by controlling the dosage of beta-catenin signaling. Nat Genet 2002; 32: 594-605.

24. Otero JJ, Fu W, Kan L, et al. Beta-catenin signaling is required for neural differentiation of embryonic stem cells. Development 2004; 131: 3545-57.

25. Ying QL, Stavridis M, Griffiths D, et al. Conversion of embryonic stem cells into neuroectodermal precursors in adherent monoculture. Nat Biotechnol 2003; 21 : 183-6.

26. Gottlieb DI. Large-scale sources of neural stem cells. Annu Rev Neurosci 2002; 25: 381-407.

27. Okada Y, Shimazaki T, Sobue G, et al. Retinoic-acid-concentration-dependent acquisition of neural cell identity during in vitro differentiation of mouse embryonic stem cells. Dev Biol 2004; 275: 124-42.

28. Ruiz I, Altaba A, Stecca B, Sanchez P. Hedgehog-Gli signaling in brain tumors: stem cells and paradevelopmental programs in cancer. Cancer Lett 2004; 204: 145-57.

29. Lang KJ, Rathjen J, Vassilieva S, et al. Differentiation of embryonic stem cells to a neural fate : a route to re-building the nervous system? J Neurosci Res 2004; 76: 184-92.

30. Mizuseki K, Sakamoto T, Watanabe K, et al. Generation of neural crest-derived peripheral neurons and floor plate cells from mouse and primate embryonic stem cells. Proc Natl Acad Sci USA 2003; 100: 5828-33.

31. Wichterle H, Lieberam I, Porter JA, et al. Directed differentiation of embryonic stem cells into motor neurons. Cell 2002; 110 : 385-97.

32. Shiotsugu J, Katsuyama Y, Arima K, et al. Multiple points of interaction between retinoic acid and FGF signaling during embryonic axis formation. Development 2004; 131 : 2653-67.

33. Altmann CR, Bell $\varepsilon$, Sczyrba A, et al. Microarray-based analysis of early development in Xenopus laevis. Dev Biol 2001; 236: 64-75.

34. Maye $P$, Becker $S$, Kasameyer $\varepsilon$, et al. Indian hedgehog signaling in extraembryonic endoderm and ectoderm differentiation in ES embryoid bodies. Mech Dev 2000; 94 : 117-32.

35. Maye $P$, Becker $S$, Siemen $H$, et al. Hedgehog signaling is required for the differentiation of $\varepsilon S$ cells into neurectoderm. Dev Biol 2004; 265: 276-90.

36. Barberi T, Klivenyi P, Calingasan NY, et al. Neural subtype specification of fertilization and nuclear transfer embryonic stem cells and application in parkinsonian mice. Nat Biotechnol 2003; $21: 1200-7$.

37. Simon HH, Bhatt L, Gherbassi D, et al. Midbrain dopaminergic neurons: determination of their developmental fate by transcription factors. Ann NY Acad Sci 2003; 991: 36-47.

38. Kim JH, Auerbach JM, Rodriguez-Gomez JA, et al. Dopamine neurons derived from embryonic stem cells function in an animal model of Parkinson's disease. Nature 2002; 418: 50-6.

39. Jiang C, Wan X, He Y, et al. Age-dependent dopaminergic dysfunction in Nurrl knockout mice. Exp Neurol 2005; 191: 154-62.

40. Chandran S, Kato H, Gerreli D, et al. FGF-dependent generation of oligodendrocytes by a hedgehog-independent pathway. Development 2003; 130: 6599-609.

41. Brustle 0, Jones KN, Learish RD, et al. Embryonic stem cell-derived glial precursors: a source of myelinating transplants. Science 1999; 285: 754-6.

42. Liu S, Qu Y, Stewart TJ, et al. Embryonic stem cells differentiate into oligodendrocytes and myelinate in culture and after spinal cord transplantation. Proc Natl Acad Sci USA 2000; 97 : 6126-31.

43. Xian HQ, McNichols $\varepsilon$, St Clair A, et al. A subset of ES-cell-derived neural cells marked by gene targeting. Stem Cells 2003; $21: 41-9$.

44. Angelov DN, Arnhold S, Andressen C, et al. Temporospatial relationships between macroglia and microglia during in vitro differentiation of murine stem cells. Dev Neurosci 1998; 20: 42-51.

45. Xian H, Gottlieb DI. Dividing Olig2-expressing progenitor cells derived from ES cells. Glia $2004 ; 47: 88-101$.

46. Gardner RL, Brook FA. Reflections on the biology of embryonic stem (ES) cells. Int J Dev Biol $1997 ; 41: 235-43$

47. Ahn JI, Lee KH, Shin DM, et al. Comprehensive transcriptome analysis of differentiation of embryonic stem cells into midbrain and hindbrain neurons. Dev Biol 2004; 265: 491-501.

48. Appel B, Eisen JS. Retinoids run rampant: multiple roles during spinal cord and motor neuron development. Neuron 2003; 40: 461-4.

49. Arnhold S, Lenartz D, Kruttwig K, et al. Differentiation of green fluorescent protein-labeled embryonic stem cell-derived neural precursor cells into Thy-l-positive neurons and glia after transplantation into adult rat striatum. J Neurosurg 2000; 93: 1026-32.

50. Strubing C, Ahnert-Hilger G, Shan J, et al. Differentiation of pluripotent embryonic stem cells into the neuronal lineage in vitro gives rise to mature inhibitory and excitatory neurons. Mech Dev 1995; $53: 275-87$.

51. Cazillis M, Gonzalez BJ, Billardon C, et al. VIP and PACAP induce selective neuronal differentiation of mouse embryonic stem cells. Eur J Neurosci 2004; 19: 798-808.

52. Kawasaki H, Mizuseki K, Nishikawa S, et al. Induction of midbrain dopaminergic neurons from ES cells by stromal cell-derived inducing activity. Neuron $2000 ; 28: 31-40$. 